\title{
Prevalence of malnutrition in outpatients with chronic obstructive pulmonary disease
}

\author{
P. F. Collins ${ }^{1}$, R. J. Stratton ${ }^{1}$, R. Kurukulaaratchy ${ }^{2}$, H. Warwick ${ }^{3}$, A. L. Cawood ${ }^{1,4}$ and M. Elia ${ }^{1}$ \\ ${ }^{1}$ Institute of Human Nutrition, School of Medicine, University of Southampton, Southampton, UK, ${ }^{2}$ Department of \\ Respiratory Medicine, Southampton University Hospital NHS Trust, Southampton SO16 6YD, UK, ${ }^{3}$ Department of Nutrition \\ and Dietetics, Southampton University Hospital NHS Trust, Southampton SO16 6YD, UK and ${ }^{4}$ Medical Affairs, Nutricia, \\ Wiltshire BA14 OXQ, UK
}

Disease-related malnutrition is common in patients with chronic obstructive pulmonary disease (COPD) and is associated with increased hospitalisation and increased mortality ${ }^{(1)}$. Despite this there remains confusion as to the exact prevalence of malnutrition in COPD outpatients, with estimates between $10 \%$ and $45 \%$ depending on the method of nutritional assessment used ${ }^{(2)}$. Using the 'Malnutrition Universal Screening Tool' 'MUST' ${ }^{\text {(3) }}$, this survey aimed to establish the local prevalence of malnutrition in outpatients with COPD.

A prospective nutritional screening survey was carried out between July 2008 and May 2009 at a large teaching hospital (Southampton General Hospital (SGH)) and a smaller community hospital within Hampshire (Lymington New Forest Hospital; LYM). Four hundred and twenty five outpatients with COPD were routinely screened with 'MUST'; 190 at SGH, 235 at LYM; 223 males, 202 females; mean age 73 (SD 9.9) years; mean BMI 25.9 (SD 6.4) $\mathrm{kg} / \mathrm{m}^{2}$. Disease severity ${ }^{(4)}$ was obtained for 246 patients and related to malnutrition risk.

Overall prevalence of malnutrition was $21 \%$ (95\% CI 17-25\%; $7 \%$ medium risk, $14 \%$ high risk) and this was two-fold greater in those with severe disease compared to those with mild and moderate disease (mild $13 \%$, moderate $12 \%$, severe $26 \% ; \chi^{2} P=0.027$ ). Age and sex were not significantly related to malnutrition risk; however, the prevalence was significantly higher at the larger teaching hospital (26\% versus $17 \%, P=0.020)$.

Malnutrition is common in COPD outpatients with an overall prevalence of $21 \%$ within the study population, the majority of which were at high risk requiring nutritional treatment. The overall prevalence of malnutrition significantly increased with disease severity. This is the first published survey to use 'MUST' in outpatients with COPD.

This work was Funded by an unrestricted educational grant from Nutricia.

1. Collins PF, Elia M, Smith TR et al. (2009) Abstract submitted to the BAPEN Conference 2009.

2. Stratton RJ, Green CJ \& Elia M (2003) Disease-Related Malnutrition: An Evidence Based Approach to Treatment. Oxford: CABI Publishing.

3. Elia M (editor) (2003) The 'MUST' Report. Redditch, Worcs.: BAPEN (http://www.bapen.org.uk).

4. National Institute for Clinical Excellence (2004) Clinical Guideline 12 